\title{
Enriching MPEG-7 User Models with Content Metadata
}

\author{
Harry Agius and Marios C. Angelides \\ Brunel University, School of Information Systems, Computing and Mathematics, \\ St John's, Uxbridge, Middlesex, UB8 3PH, UK \\ \{harryagius, angelidesm\}@acm.org
}

\begin{abstract}
MPEG-7 is an XML-based standard that provides tools for creating rich and structured multimedia content metadata. However, only an extremely limited range of preferences can be specified for user models and multimedia content metadata created by other parts of the standard cannot be fully exploited. This results in a very incomplete mapping of user models to content models. We present an approach to address the problem by representing user models by means of existing MPEG-7 content description tools.
\end{abstract}

\section{Introduction}

User modelling in MPEG-7 is encompassed by means of user preferences and usage histories $[1,2]$. The former may be expressed for types of content, ways of browsing content and ways of recording content, while the latter may be expressed as lists of user actions within applications. Initially, this appears very inclusive; however, closer inspection reveals significant limitations. Having created a wealth of multimedia content metadata, only an extremely limited range of user preferences may relate directly to it, thus making it impossible to fully exploit it. For example, where metadata regarding events and objects present in a media stream exist, it is not possible to specify user preferences for them, nor user preferences for their relationships with other events and objects. At best, only keywords can be represented and then only for creation preferences. The upshot is a user model that is far removed from the content model.

One solution is to use the MPEG-7 DDL (Description Definition Language) to modify and add to the existing MDS (Multimedia Description Schemes) to expand its scope and abilities. The drawback of this is that it renders the representation incompatible with existing parsers and validation tools without modification. We have therefore been experimenting with an alternative approach. By considering the user model to be isomorphic to the multimedia content model, the user model may then be represented by means of existing MPEG-7 content description tools. This enables direct overlaying of the user model with the content model, permitting easier and more transparent mapping between the two.

The next section examines MPEG-7 user modelling in further detail. Then Section 3 introduces a 'hanging basket' model which is used as the underlying basis for structuring the MPEG-7 content metadata in Section 4. Section 5 shows how the user model may also be represented by the 'hanging basket' model, which is then mapped onto MPEG-7 user preference metadata in Section 6. Section 7 concludes the paper.

\section{User modelling in MPEG-7}

The UserPreferences DS (Description Scheme) is shown in Figure 1. It enables relative user preferences in the range $[-100,100]$ to be specified for: creation, classification and source content types using the FilteringAndSearchPreferences DS; content browsing using the BrowsingPreferences DS, which may be conditioned on certain times, locations and genres, and; content recording using the RecordingPreferences DS, which may contain one or more RecordingRequests [1, 2]. The UsageHistory DS (Figure 2) describes a set of UserActionHistory elements, which in turn describe a set of UserActionList elements, each of which contains UserAction elements. Identifiers for applications associated with each action may be included as well as the action times and the time extents of the consumed content. References to associated content descriptions may also be used [1].

A body of recent research has investigated the application of MPEG-7 user modelling tools. For TV and broadcasting, approaches have ranged from asking users to fill out a form specifying a small number of preferences, such as genre and channels of interest [3], 
to using fuzzy inferencing to construct and update user preferences regarding genre, language and broadcast channel based on usage history [4].

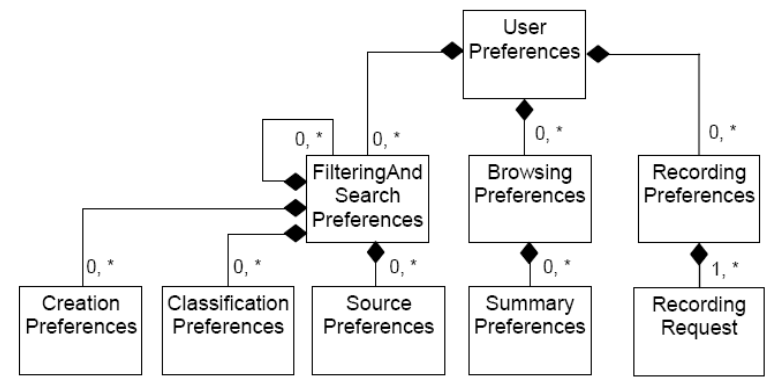

Figure 1. UserPreferences DS in MPEG-7 [2].

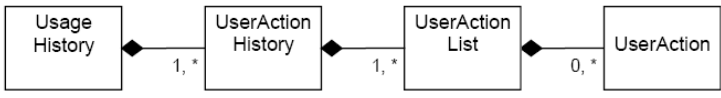

Figure 2. UsageHistory DS in MPEG-7 [1].

Taking clients and networks into account, Martínez et al. [5] use ClassificationPreferences and CreationPreferences for filtering content, after which SourcePreferences are used for finding a suitable content variation, while Steiger et al. [6] acquire initial prioritisation of user preferences for categories such as content topic, language and source via a form, which is then stored using the UserPreferences DS and updated automatically via the UsageHistory DS by giving higher priority to content categories that are often selected or by adding them to the initial preferences.

For personalised video summaries, Tseng et al. [7] use MPEG-7 and MPEG-21 user preferences and user time constraints to output an optimised set of selected video segments. User preferences include category, channel and default viewing time. Further, nonpersistent, preferences are then specified as a query.

Some researchers have sought to extend the MPEG7 MDS to enhance user modelling capabilities. Jin et al. [8] extend the UserPreferences DS so that 'events' may be accommodated within the ClassificationPreferences DS and suitable broadcast programming recommended according to suitable matching events in the content metadata. More substantially, Tsinaraki and Christodoulakis [9] define SCreationPreferencesType as a subtype of the existing CreationPreferencesType and introduce a set of content preference description elements of type WeighedSemanticBagType, an extension of the existing SemanticBagType with a preferenceValue attribute. To allow semantic preferences related to multimedia summaries, they also define
SSummaryPreferencesType as a subtype of the existing SummaryPreferencesType, which specifies a set of semantic summary preference descriptions.

\section{3. 'Hanging basket' content model}

This section formally defines an underlying model of content metadata for media streams, which can be directly mapped onto an MPEG-7 content model and subsequently an MPEG-7 user model. Figure 3 provides a visual illustration of the model, whose shape is similar to that of a hanging basket.

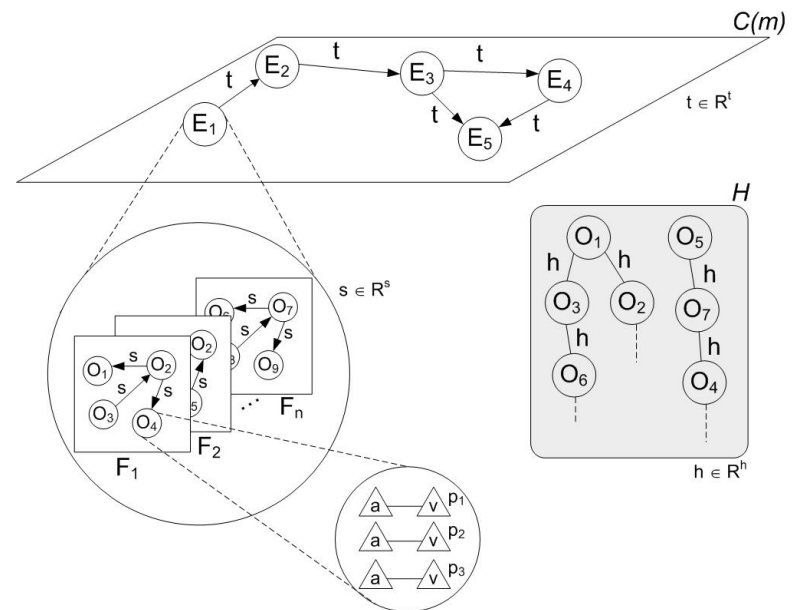

Figure 3. 'Hanging basket' content model.

Definition 1 (Content model). Let $\mathcal{E}$ be a set of events unfolding over time within a multimedia stream $m$. Then a content model $C(m)$ is a simple, weakly connected, labelled digraph:

$$
C(m)=(\mathcal{E}, T)
$$

where $T \subset \mathcal{E} \times \mathcal{E} \times R^{t}$ and $R^{t}$ is any set of binary temporal relationships.

Definition 2 (Event). An event $E \in \mathcal{E}$ is a set of weakly connected, labelled multidigraphs $F_{i}$, each of which represents spatial relationships between objects at time $i$ :

$$
E=\left\{F_{i}: F_{i}=\left(\mathcal{O}_{i}, S_{i}\right)\right\}
$$

where $\mathcal{O}_{i}$ is a set of objects unfolding within $E$ at time $i, S_{i} \subset \mathcal{O}_{i} \times \mathcal{O}_{i} \times R^{s}$ and $R^{s}$ is any set of binary spatial relationships. 
Definition 3 (Object). An object $O \in \mathcal{O}$ is a set of properties, where each property $p_{i}$ describes the object and is specified as an attribute-value pair:

$$
O=\left\{p_{i}: p_{i}=(a, v)_{i}\right\}
$$

where $v$ is a value for attribute $a$.

Definition 4 (Object hierarchy). An object hierarchy $H$ is a simple, labelled digraph:

$$
H=(\mathcal{O}, \Gamma)
$$

where $\Gamma \subset \mathcal{O} \times \mathcal{O} \times R^{h}$ and $R^{h}$ is any set of binary hierarchical relationships.

\section{Mapping the 'hanging basket' to an MPEG-7 content model}

To map the 'hanging basket' model to an MPEG-7 content model, we follow COSMOS-7 [10] and use two MPEG-7 top-level types: the SemanticDescriptionType to group the events and temporal relationships as specified in (1), the objects and object properties specified in (3) and the object hierarchy in (4), and the ContentEntityType to group the spatial relationships specified in (2).

The Semantic DS and Event DS are used to represent events as shown in Figure 4. One or more events are grouped using the Semantic DS which enables related events, such as those related to specific objects, to be proximate. Each event uses several elements within the Event DS. The MedialnformationRef element within the MediaOccurrence DS is used to refer to the appropriate video segment where TemporalMaskTypes define appropriate masks on the segment. Events are related to objects through the use of the SemanticRelation CS (Classification Scheme) and the agent relation. CSs are a type of DS that define a set of standard terms for a domain. Temporal relationships between events are grouped using the Semantic DS while the relationships themselves are represented via the Graph DS, with each temporal relationship defined using the TemporalRelation CS.

Each object is grouped with its properties using the Semantic DS as illustrated in Figure 5. Objects are related to events through the use of the SemanticRelation CS and the agentOf relation (the inverse of the agent relation). The Object DS is used to represent the objects. It includes elements describing the composition of an object from sub-objects. The MediaOccurrence elements (together with
TemporalMasks) are used as previously described to relate specific video segments that reflect the occurrences of objects. The SemanticState DS represents the object properties. To enable MediaOccurrences to be related to specific object properties, each property is modelled as a separate SemanticState. It is related to a specific object using a Relation element of type propertyOf. The AttributeValuePair element is used to specify the properties themselves. No restrictions are made on which properties may be modelled, so long as they conform to the given structure. Objects are related to each other in the object hierarchy through the use of the SemanticRelation CS. The Semantic DS is used to group all object relationships together with a graph representing the relationships through the specializes relation (the inverse relation is generalizes).

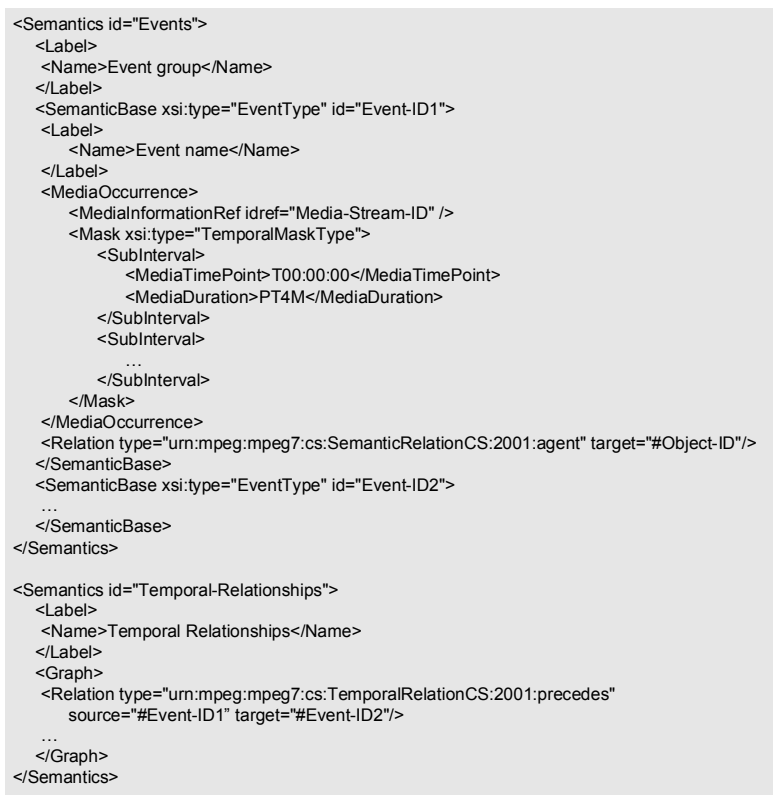

Figure 4. Events in MPEG-7.

Spatial relationships are specified on a per video stream basis, as shown in Figure 6. The video is segmented so that spatial-relationship inheritance may be deployed, whereby child segments inherit a parent segment's spatial relationships as well as specifying their own spatial relationships. The VideoSegment DS is used to create segments using the VideoSegmentTemporalDecompositionType and the VideoSegmentSpatioTemporalDecompositionType.

MediaTime elements are used to delineate the segments. The MovingRegion DS is used to identify objects as regions within the video which are related to the content-modelled objects specified in the previous section through the use of the SemanticRef element (a Reference data type) that the VideoSegment DS 
inherits from the Segment DS in the MDS. The spatial relationships themselves are specified using the SpatialRelation CS through the Graph DS.

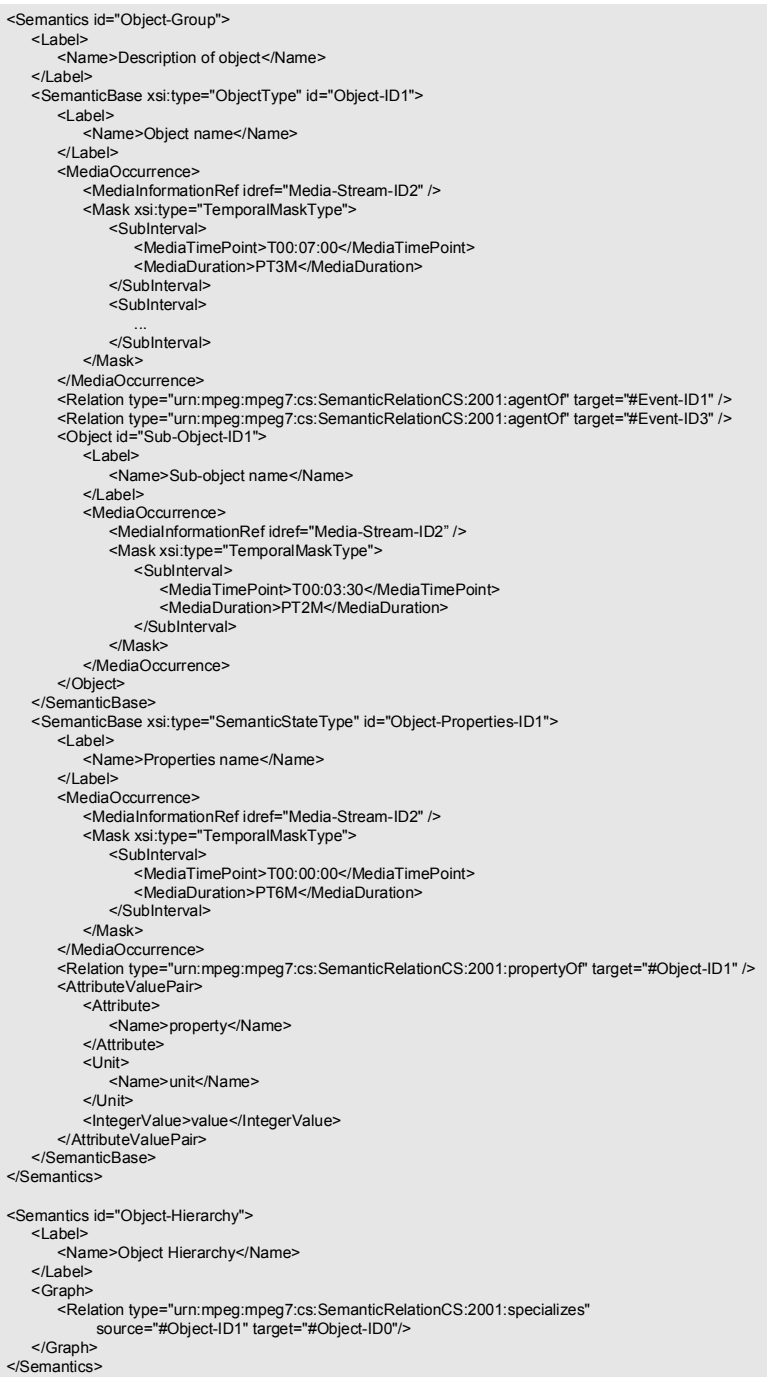

Figure 5. Objects in MPEG-7.

\section{A user model isomorphic to the 'hanging basket' content model}

Having defined the 'hanging basket' content model and its representation in MPEG-7, it is now possible to define the user model. The user model is defined as a weighted version of the content model and therefore is isomorphic to it. In this context, the entities defined in Section 3 become 'preferred' in the sense that they are involved in the expression of preferences.

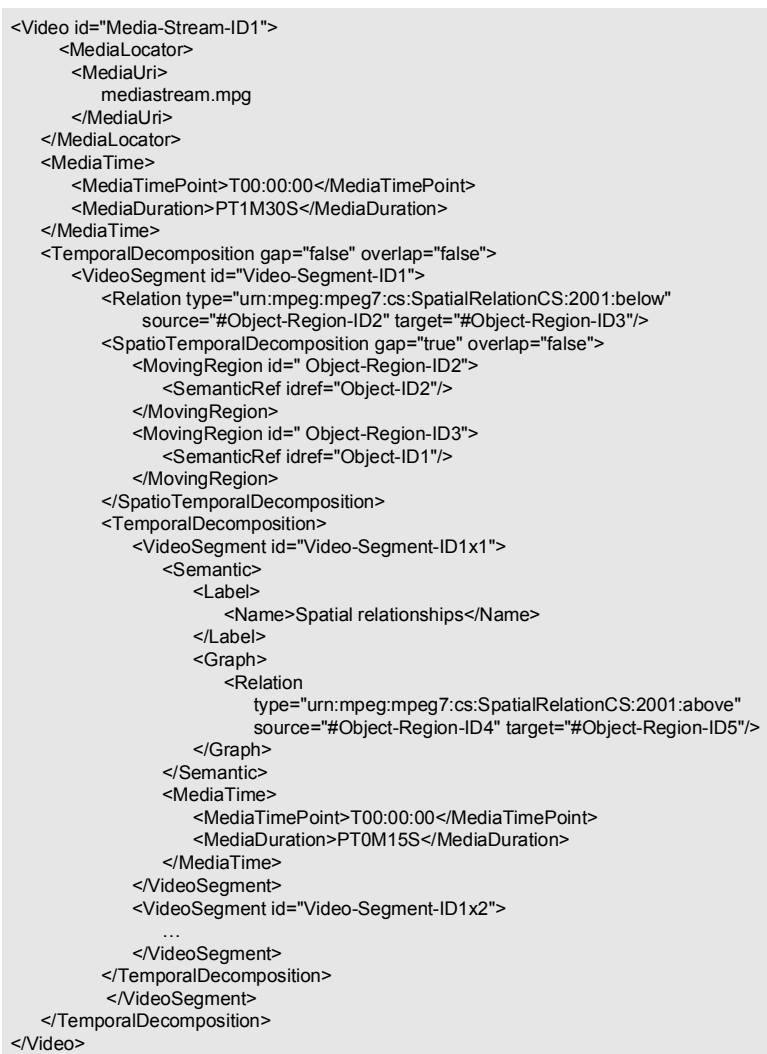

Figure 6. Spatial relationships in MPEG-7.

Definition 5 (User model). For a multimedia stream $m$, a user model $U(m)$ is a simple, weakly connected, labelled, weighted digraph:

$$
U(m)=\left(\mathcal{E}^{\prime}, T^{\prime}\right)
$$

with temporal relationship preference weights $w^{t}: T^{\prime} \rightarrow \mathbb{R}$ and event preference weights $w^{e}: \mathcal{E}^{\prime} \rightarrow \mathbb{R}$, where $\mathcal{E}^{\prime}$ is the set of preferred events and $T^{\prime} \subset \mathcal{E}^{\prime} \times \mathcal{E}^{\prime} \times R^{t}$. Where the user has no preference, the weight is zero or another value predefined as neutral. Thus, $U(m)$ is a node- and arcweighted version of $C(m)$ as defined in (1). Hence,

$$
U(m) \cong C(m)
$$

Definition 6 (Preferred event). A preferred event $E^{\prime} \in \mathcal{E}^{\prime}$ is a set of weakly connected labelled multidigraphs $F_{i}^{\prime}$, each representing preferred spatial relationships between preferred objects at time $i$ :

$$
E^{\prime}=\left\{F_{i}^{\prime}: F_{i}^{\prime}=\left(\mathcal{O}_{i}^{\prime}, S_{i}^{\prime}\right)\right\}
$$


with spatial relationship preference weights $w^{s}: S_{i}^{\prime} \rightarrow \mathbb{R}$ and object preference weights $w^{o}: \mathcal{O}_{i}^{\prime} \rightarrow \mathbb{R}$ for each $F_{i}^{\prime} \in E^{\prime}$, where $\mathcal{O}_{i}^{\prime}$ is the set of preferred objects unfolding within $E^{\prime}$ at time $i$ and $S_{i}^{\prime} \subset \mathcal{O}_{i}^{\prime} \times \mathcal{O}_{i}^{\prime} \times R^{s}$. Thus, $F_{i}^{\prime}$ is a node- and arcweighted version of $F_{i}$ as defined in (2). Hence,

$$
F_{i}^{\prime} \cong F_{i}
$$

Definition 7 (Preferred object). A preferred object $O^{\prime} \in \mathcal{O}^{\prime}$ is a set of preferred properties, where each property $p_{i}^{\prime}$ describes the object and is specified as an attribute-value-preference triple:

$$
O^{\prime}=\left\{p_{i}^{\prime}: p_{i}^{\prime}=(a, v, \pi)_{i}\right\}
$$

where $\pi$ is a preference for the pairing of $a$ and $v$.

Definition 8 (Preferred object hierarchy). A preferred object hierarchy $H^{\prime}$ is a simple, labelled digraph:

$$
H^{\prime}=\left(\mathcal{O}^{\prime}, \Gamma^{\prime}\right)
$$

where $\Gamma^{\prime} \subset \mathcal{O}^{\prime} \times \mathcal{O}^{\prime} \times R^{h}$. Hence,

$$
H^{\prime} \cong H
$$

The expression of user preferences for the object hierarchy is not encompassed within the above definition since it is not meaningful for a user to hold a preference for an object generalisation or specialisation, e.g. a user may prefer mammals or elephants but not elephants which are specialisations of mammals since this is always the case.

\section{A content-enriched MPEG-7 user model}

Since the user model is isomorphic to the 'hanging basket' content model, its representation in MPEG-7 utilises similar description tools to those used in Section 4. The Relation DS, which was used extensively throughout the 'hanging basket' content model, employs a strength attribute which is of type zeroToOne. Consequently, all preferences must be specified as a float number in the range $[0,1]$, rather than the range $[-100,100]$ used by the preferenceValue datatype within the UserPreferences DS and UsageHistory DS. Consequently, within the $[0,1]$ range, 0.5 is taken as the neutral (default) value and values below this specify negative preferences (dislikes), while values above it specify positive preferences (likes). We now describe the key description tools employed and the structure of the user model in MPEG-7, which is shown in Figure 7.

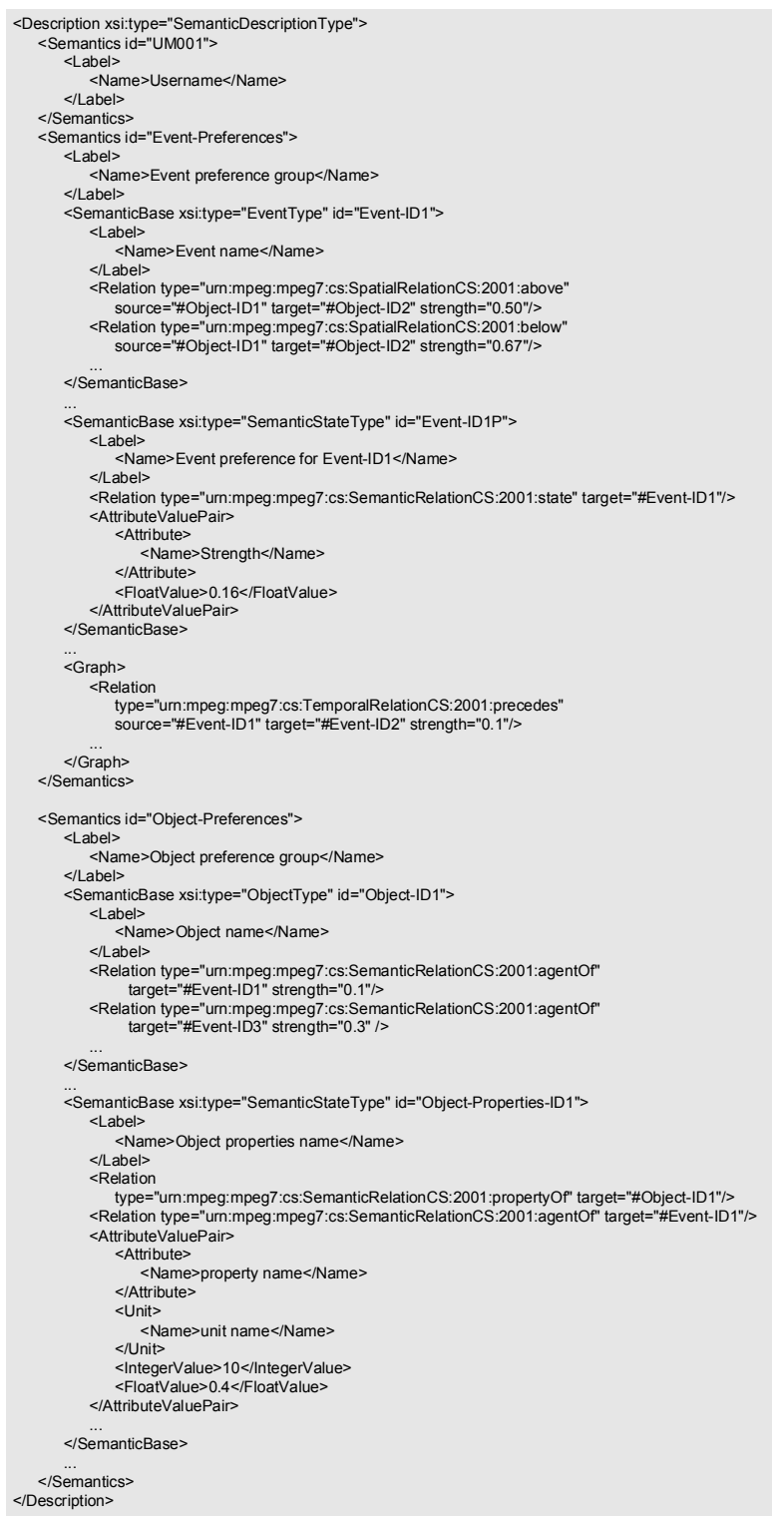

Figure 7. User model in MPEG-7.

A single top-level type is used, the SemanticDescriptionType, to gather together an entire user model for a single user. The preferences within the model are then split into two parts.

In the first part, the Semantic DS is used to group preferred events and preferred temporal relationships as specified in (5) and preferred spatial relationships between objects as specified in (7). The Event DS is used to express the latter so that spatial relationship preferences are specified via the SpatialRelation CS 
within the context of the encompassing event. The strength attribute of the Relation element is used to express the user's preference for the spatial positioning of two objects within the event. The SemanticState DS is used to express the user's preference for an event as an AttributeValuePair. The state relation from the SemanticRelation CS is used to tie the preference with the relevant Event DS. Finally, preferred temporal relationships are specified using the Graph DS, as previously, with the addition of the strength attribute within each Relation element.

In the second part, the Semantic DS is used to group preferred objects and preferred object properties as specified in (9). The Object DS encapsulates preferences for an object within an event via one or more Relation elements of type agentOf appended with the strength attribute. The SemanticState DS is then used to express the user's preference for the properties of an object. The propertyOf relation from the SemanticRelation CS is used to tie the set of preferences with the relevant Object DS, while the agentOf relation is used to define within which event the preferred object properties are expressed for. The preferences are then specified as sets of AttributeValuePairs where the Attribute, Unit and initial value correspond to the content model while the final FloatValue expresses the user's preference.

For the preferred object hierarchy specified in (10), the user model assumes the object hierarchy from the content model due to (11).

\section{Conclusion}

User modelling in MPEG-7 through the UserPreferences DS and UsageHistory DS is limited; specifically in terms of the range of user preferences that can be specified and in relating those preferences to content metadata. One solution is to represent the user model using existing MPEG-7 content description tools which enables direct overlaying of the user model with the content model so that user preferences can be expressed for the entities and relationships of the content model. We have shown how such a solution can be achieved through the formal definition and MPEG-7 representation of 'hanging basket' content and user models. The MPEG-7 user model expresses preferences through the strength attribute of the Relation DS and a FloatValue within the AttributeValuePair element. This is not the only possible representation however and future research work will look at alternatives, such as matrices within multiple SemanticState DSs. We are also interested in examining how existing user description tools may be used to complement the representation.

\section{References}

[1] ISO/IEC, "Information Technology - Multimedia Content Description Interface - Part 5: Multimedia Description Schemes," Geneva, Switzerland, International Standard 15938-5, 2003.

[2] ISO/IEC, "Information Technology - Multimedia Content Description Interface - Part 5: Multimedia Description Schemes: Amendment 2: Multimedia Description Schemes User Preference Extensions," Geneva, Switzerland, International Standard 159385/Amd.2, 2005.

[3] M. Rovira, J. González, A. López, J. Mas, A. Puig, J. Fabregat, and G. Fernàndez, "IndexTV: A MPEG-7 Based Personalized Recommendation System for Digital TV," Proceedings of 2004 IEEE International Conference on Multimedia and Expo, Vol. 2, Taipei, Taiwan, 27-30 June, 2004, pp. 823-826.

[4] A. M. Ferman, P. van Beek, J. H. Errico, and M. I. Sezan, "Multimedia Content Recommendation Engine with Automatic Inference of User Preferences," Proceedings of 2003 IEEE International Conference on Image Processing, Vol. 3, Barcelona, Catalonia, Spain, 14-17 September, 2003, pp. 49-52.

[5] J. M. Martínez, C. González, O. Fernández, C. Garcia, and J. de Ramón, "Towards Universal Access to Content using MPEG-7," Proceedings of 10th ACM International Conference on Multimedia, Juan-lesPins, France, 1-6 December, 2002, pp. 199-202.

[6] O. Steiger, T. Ebrahimi, and D. M. Sanjuán, "MPEGBased Personalized Content Delivery," Proceedings of 2003 IEEE International Conference on Image Processing, Vol. 3, Barcelona, Catalonia, Spain, 14-17 September, 2003, pp. 45-48.

[7] B. L. Tseng, C.-Y. Lin, and J. R. Smith, "Using MPEG-7 and MPEG-21 for Personalizing Video," IEEE Multimedia, vol. 11, no. 1, pp. 42-53, 2004.

[8] S. H. Jin, T. M. Bae, Y. M. Ro, H.-R. Kim, and M. Kim, "Intelligent Broadcasting System and Services for Personalized Semantic Contents Consumption," Expert Systems with Applications, vol. 31, pp. 164-173, 2006.

[9] C. Tsinaraki and S. Christodoulakis, "A Multimedia User Preference Model that Supports Semantics and its Application to MPEG 7/21," Proceedings of 12th International Multi-Media Modelling Conference, Beijing, China, 4-6 January, 2006.

[10] M. C. Angelides and H. Agius, "An MPEG-7 Scheme for Semantic Content Modelling and Filtering of Digital Video," ACM/Springer Multimedia Systems, vol. 11, no. 4, pp. 320-339, 2006. 